\title{
A TAPHONOMIC ANALYSIS OF THE FAUNAL ASSEMBLAgE FROM YAK CAMP ROCKSHELTER, S.E. CAPE YORK PENINSULA
}

\author{
BERNARD M.J. HUCHET
}

Archaeology of Palaeosnthropology

The Uaivergity of New Bagland

\section{INTRODUCTION}

This paper presents the results of a taphonomic analysis of a part of the faunal assemblage recovered from Yam Camp shelter, a prehistoric Aboriginal site located on shepherd Creek, a tributary to the little Laura River, S.E. Cape York Peninsula. The site was excavated in July 1989 under the directorship of Dr. Mike Morwood, Department of Archaeology and Paleoanthropology, The University of New England. Some aspects of the archaeology of this site have already been discussed by Morwood (1989; 1990) and Pearson (1989) and a full excavation report will be published in due course. Due to the extremely fragmented nature of the faunal assemblage, a detailed reconstruction of the taphonomic history was considered necessary prior to the study of economic patterns at the site.

More specifically, this study aims to establish not only the agents involved in the deposition of bones but also those responsible for the fragmentation of the faunal assemblage. These aims are achieved by considering a range of data including the length and shape of the bone fragments, types of breakage, and marks represented on the bones. From the results it is inferred that the bulk of the assemblage was accumulated as a result of human behaviour, that the high degree of bone fragmentation is the result of chemical deterioration that trampling may have accelerated the fragmentation process and that, although bone was introduced into the matrix by non-human predators, the amount was not substantial.

The bone sample analysed for this study was recovered from one of five $1 \mathrm{~m} \times 1 \mathrm{~m}$ excavation trenches (Square J5). The square was subdivided into four $50 \mathrm{~cm} \times 50 \mathrm{~cm}$ quadrants $(J 5 a-5 d)$ for excavation control. It was excavated in arbitrary units (spits). Bedrock was reached at a depth of $1.15 \mathrm{~m}$. Excavation revealed two clearly differentiated stratigraphic units as follows:

Layer 1 (LI): This is the uppermost layer (22-24cm) which is composed of very fine ashy sediment relatively free of rockfall ( $\mathrm{pH}=8.5$ ). It may be subdivided into two sub-layers. The topmost, L1a, represents a treadage layer, is about $7-8 \mathrm{~cm}$ thick and is grittier and less compact than the lower sub-layer, Llb. Ll corresponds to Spits 1 to 7 . This layer contained the bulk of the faunal material. 
Layer 2 (L2) This is the lower part of the deposit $(24-115 \mathrm{~cm})$ which may be distinguished from $I 1$ by the presence of large boulders (up to $1 \mathrm{~m}$ diam.) as well as a profusion of smaller rocks, all of which probably derive from rock falls. The matrix is composed of coarse sediments derived from the decomposition of sandstone. Very little charcoal. was present. The $\mathrm{pH}$ was 7.0 .

\section{Preservation}

In order to understand site formation as well as post-depositional processes, it is useful to discuss the vertical distribution of archaeological materials. These data are provided in Table 1 and indicate that changes in the frequency of bone, shell, charcoal and other organics correlate with those for stone artefacts. This correspondence suggests that preservation of biological remains was excellent at Yam Camp. If this, were not the case, one would expect differential preservation of these components (for example, shell would be less represented with increasing age of deposit in comparison to charcoal and bone). At Yam Camp, all components are present throughout the sequence except for shell which is lacking in the lowermost spits, a fact more likely due to low discard frequency than preservation factors.

Two other lines of evidence support this claim of uniformly excellent preservation. First, the $\mathrm{pH}$ values are 8.5 and 7.0 in $\mathrm{L} 1$ and $\mathrm{L} 2$ respectively. Second, the data for bone preservation indicate that the process of bone decomposition, if operating at all, operated at a uniform rate with deposit depth and did not result in a significant loss of faunal material. The frequencies for four categories of edge-sharpness remains relatively constant through the deposit. If significant bone decomposition were operating at Yam Camp, one would expect a change in edge-sharpness from sharp to eroded through the sequence from top to bottom. Table 2 shows that the "sharp" category remains dominant over the "eroded" category at Yam Camp. Thus, if natural destruction of bones was taking place it did not significantly affect this assemblage and did not lead to differential preservation through time.

Another means of assessing differential preservation processes relies on the premise that bone preservation potential increases with the intensity to which it has been subjected to fire. In cases where the frequency of unburnt bone decreases with deposit depth relative to that of burnt and calcined bone, it is inferred that natural processes resulted in differential destruction (see Aplin and Gollan 1982). In cases of extreme decomposition, only calcined bone is expected to survive. However, as pointed out by Burke (1988:72), a major limitation of this approach is that the ratio of burnt to unburnt bone can be affected by changes in the frequency of hearth construction at a site. This problem was encountered at Yam Camp where an increase in the frequency of charred (i.e. burnt plus calcined) bone in spits 5 and 6 is associated with an increase in charcoal and ash which is considered to be hearth-derived (Table 3). In the four uppermost spits, hearth remains cover a surface area of about $15 \%$ (average); however, in spits 5 and 6 this area increases to about 85\%. Also, the low frequency of charred bone in deposits below spit 6 correlates with a low density of charcoal and ash. Thus, the data concerning bone-charring at Yam Camp is perhaps better suited to assessment of hearth construction intensity than bone decay rate. 
Table 1. Distribution of archaeological remains, Yam Camp J5a* .

\begin{tabular}{|c|c|c|c|c|c|c|c|}
\hline \multirow[t]{2}{*}{$\bar{L}$} & QUADRANT & \multirow{2}{*}{$\begin{array}{c}\text { CHARCOAL } \\
\text { (g) }\end{array}$} & \multirow{2}{*}{$\begin{array}{l}\text { ORGANICS } \\
\text { (g) }\end{array}$} & \multirow{2}{*}{$\begin{array}{l}\text { BONE } \\
(g)\end{array}$} & \multirow{2}{*}{$\begin{array}{c}\text { SHELL } \\
\text { (g) }\end{array}$} & \multicolumn{2}{|c|}{ STONE } \\
\hline & \& SPIT & & & & & (g) & (No) \\
\hline & $J 5 a / 1$ & 108.7 & 26.0 & 7.7 & - & 3.4 & 20 \\
\hline & 12 & 82.0 & 75.1 & 2.2 & - & 7.1 & 21 \\
\hline & $/ 3$ & 116.9 & 27.7 & 2.6 & - & 27.0 & 24 \\
\hline \multirow[t]{4}{*}{ L1 } & 14 & 116.5 & 12.3 & 9.2 & + & 14.2 & 48 \\
\hline & $/ 5$ & 50.9 & 17.8 & 4.8 & - & 32.4 & 42 \\
\hline & 16 & 20.4 & 6.7 & 3.0 & - & 27.6 & 47 \\
\hline & 17 & 10.8 & 3.8 & 0.1 & - & 4.6 & 10 \\
\hline & $/ 8$ & 12.0 & 3.7 & 0.1 & - & 1.1 & 5 \\
\hline & 19 & 0.7 & 5.7 & - & - & 0.1 & 1 \\
\hline & 110 & 0.1 & 5.5 & - & - & - & - \\
\hline & $/ 11$ & 0.3 & 0.9 & - & - & - & - \\
\hline & $/ 12$ & 0.1 & 0.8 & - & - & 0.2 & 1 \\
\hline \multirow[t]{6}{*}{ L2 } & $/ 13$ & 1.0 & - & - & - & 31.1 & 4 \\
\hline & $/ 14$ & 0.1 & 0.1 & - & - & - & - \\
\hline & $/ 15$ & 0.1 & 0.1 & - & - & - & - \\
\hline & $/ 16$ & 1.8 & $4 \cdot 3$ & 0.1 & - & 2.4 & 2 \\
\hline & $/ 17$ & 0.1 & 0.5 & - & - & - & - \\
\hline & $/ 18$ & 0.3 & 0.3 & - & - & 0.2 & 1 \\
\hline
\end{tabular}

+ shell present in trace only

J5d data not represented as the pattern is repeated.

Table 2. Edge characteristics of bones from Yam Camp J5a.

\begin{tabular}{|c|c|c|c|c|c|c|c|c|c|}
\hline \multirow[t]{2}{*}{ SPIT } & \multicolumn{2}{|c|}{ SHARP } & \multicolumn{2}{|c|}{ SMOOTH } & \multicolumn{2}{|c|}{ ROUNDED } & \multicolumn{2}{|c|}{ ERODED } & \multirow{2}{*}{$\begin{array}{c}\text { SAMPLE } \\
\mathbf{N}\end{array}$} \\
\hline & N & $(8)$ & $\mathbf{N}$ & $(8)$ & $\mathbf{N}$ & (8) & $\mathrm{N}$ & (8) & \\
\hline$\overline{1}$ & 48 & $(57.2)$ & 17 & $(20.2)$ & 10 & $(11.9)$ & 9 & $(10.7)$ & 84 \\
\hline 2 & 22 & $(61.1)$ & 11 & $(30.6)$ & 3 & $(8.3)$ & - & - & 36 \\
\hline 3 & 18 & $(72.0)$ & - & - & 6 & $(24.0)$ & 1 & $(4.0)$ & 25 \\
\hline 4 & 93 & $(72.7)$ & 16 & $(12.5)$ & 13 & $(10.1)$ & 6 & $(4.7)$ & 128 \\
\hline 5 & 16 & $(50.0)$ & 10 & $(31.2)$ & 6 & $(18.8)$ & - & - & 32 \\
\hline 6 & 26 & $(65.0)$ & 11 & $(27.5)$ & 2 & $(5.0)$ & 1 & $(2.5)$ & 40 \\
\hline 7 & 3 & $(37.5)$ & 3 & $(37.5)$ & 1 & $(12.5)$ & 1 & $(12.5)$ & 8 \\
\hline TOT & 266 & $(64.0)$ & 68 & $(19.3)$ & 41 & $(11.6)$ & 18 & $(5.1)$ & 353 \\
\hline
\end{tabular}

Table 3. Frequency of burnt, unburnt and calcined bone from Yam Camp J5a.

\begin{tabular}{|c|c|c|c|c|c|c|c|}
\hline \multirow[t]{2}{*}{ SPIT } & \multicolumn{2}{|c|}{ UNBURNT } & \multicolumn{2}{|c|}{ BURNT } & \multicolumn{2}{|c|}{ CALCINED } & \multirow{2}{*}{$\begin{array}{c}\text { SAMPLE SIZE } \\
N\end{array}$} \\
\hline & $\mathrm{N}$ & $(8)$ & & $(8)$ & $\mathbf{N}$ & $(8)$ & \\
\hline 1 & 82 & $(85.4)$ & 9 & $(9.4)$ & 5 & $(5.2)$ & 96 \\
\hline 2 & 43 & $(84.3)$ & 2 & $(3.9)$ & 6 & $(11.8)$ & 51 \\
\hline 3 & 35 & $(83.3)$ & 4 & $(9.5)$ & 3 & $(7.2)$ & 42 \\
\hline 4 & 153 & $(81.4)$ & 6 & $(3.2)$ & 29 & $(15.4)$ & 188 \\
\hline 5 & 35 & $(68.6)$ & 2 & $(3.9)$ & 14 & $(27.5)$ & 51 \\
\hline 6 & 26 & $(65.0)$ & 2 & $(5.0)$ & 12 & $(30.0)$ & 40 \\
\hline $7-18$ & 8 & $(89.9)$ & - & - & 1 & $(11.1)$ & 9 \\
\hline
\end{tabular}




\section{Vertical Displacement}

The presence of a $7-8 \mathrm{~cm}$ thick treadage layer at Yam Camp suggested that vertical displacement of archaeological material probably occurred inside the shelter. Thus, an attempt was made to conjoin lithic material from J5a and $J 5 d$ in order to assess the maximum depth of displacement in various parts of the sequence. several conjoins were refitted and the distribution of least-represented raw material categories were analysed. Results suggest that displacement in $\mathrm{L} 1$ did not exceed $7-8 \mathrm{~cm}$ (the thickness of the treadage layer as observed in the field). Also, movement appears to have been primarily downwards. In I2 vertical displacement does not appear to exceed $4 \mathrm{~cm}$, albeit this result needs to be confirmed using a larger artefact sample. In sum, only a small percentage of material appears to have been displaced.

\section{TAPHONOMIC ANALYSIS}

\section{Length of Bones}

Precise measurement of bones can provide information about likely agents of deposition and about processes resulting in bone breakage. Accordingly, measurements of bone length were recorded to the nearest millimetre. Lenqth refers to the maximum dimension in any one plane. Results are given in Figure 1 in $3.0 \mathrm{~mm}$ categories for a sample of 354 bones and bone pieces. These data of bone length demonstrate that the assemblage is generally represented by very small pieces of bone (virtually all bone is represented by fragments). The first step was to establigh whether or not these bone fragments were deposited as a result of carnivores defecating inside the shelter. As Dingoes are very common in the region today (Abel 1989) and since they frequently use rockshelters as dens, this proposition was carefully considered.

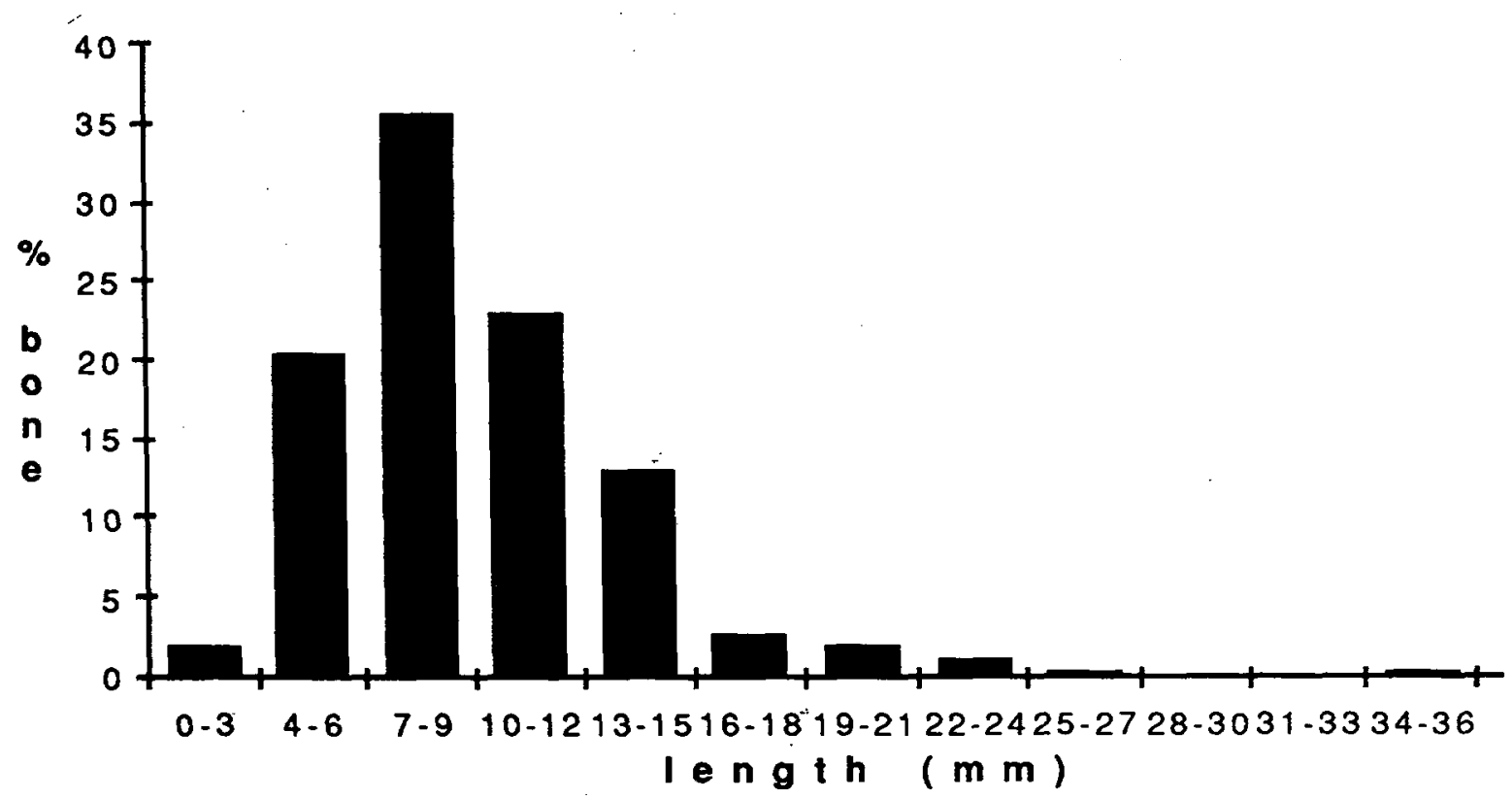

Figure 1. Iength of Yam Camp Bones. 
The only published work regarding the size of bones present in dingo scats is that of Solomon (1985:88), who carried out experiments by feeding large macropod carcasses (W. bicolor, , robustus and r. giganteus) to dingoes held in captivity. The bones were recoyered from the scats and ordered according to size class $(e . g .2 .5 \mathrm{~mm}, 5 \mathrm{~mm}$, $10 \mathrm{~mm}$, $15 \mathrm{~mm})$. Solomon's results are given in Table 4. All but one of the bones in her sample $(\mathrm{N}=840$ ) measured less than 30mm (Solomon 1985:105).

Table 4. Length of bones defecated by experimentally fed Dingoes (from solomon $1985: 58$ )

\begin{tabular}{lc}
\hline SIZE CATEGORY & \& OF SAMPLE \\
\hline $2.5 \mathrm{~mm}$ & 12.0 \\
$5.0 \mathrm{~mm}$ & 28.0 \\
$10.0 \mathrm{~mm}$ & 38.0 \\
$15.0 \mathrm{~mm}$ & 12.0 \\
$20.0 \mathrm{~mm}$ and over & 10.0 \\
\hline
\end{tabular}

As Solomon's method of measurement differs from that employed in this study, it is somewhat cumbersome to compare the two data.sets. Nevertheless, there is a close similarity between. Solomon's data and those recorded for Yam Camp, both in terms of size range and frequency within size categories. Does this mean all or most of the bones from Yam Camp were deposited by dingoes? In order to answer this question it was necessary to consider three aspects: solomon's experimental methods, breakage patterns of long bones and the shape of all bones. These are now considered in some detail.

Solomon (1985:88) fed dingoes with portions of swamp wallabies and kangaroos, animals that are generally outside the size range of animals normally preyed upon by dingoes. Wild dingoes generally hunt animals up to size of small macropods, such as hare-wallabies, which weigh about 4 to $5 \mathrm{~kg}$. Occasionally they take larger prey when hunting in packs (for review of feeding habits, see solomon 1985: Chapter 6). As a result, it is probable that bones of these smaller prey animals are more fragmented than those in Solomon's sample.

This notion is supported by evidence gathered from my analysis of two wild dingo scats collected by Malcolm Abel just above the Yam Camp rockshelter. The faeces contained the remains of at least two animals, one a possum (or marsupial of similar size), the other a small rock wallaby. An attempt was made to recover all of the bone contained in the scats by gently separating the matrix under runiing water and catching the residue in two nested geological sieves (1.0mm and $0.3 \mathrm{~mm}$ mesh). In this manner a total of 236 complete and fragmented pieces of bone were recovered. I infer from the size distribution of the bone from this sample (Figure 2) that dingoes that feed on animals of a maximum size of 4 to $5 \mathrm{~kg}$ defecate bones that are significantly smaller than those produced by dingoes fed on larger macropods. In the case of the Yam Camp dingo assemblage, over two-thirds of the bones measure $6 \mathrm{~mm}$ or less whereas in solomon's sample, two-thirds measure between approximately $5 \mathrm{~mm}$ and $10 \mathrm{~mm}$. Further, with the exception of one bone measuring $22 \mathrm{~mm}$, none of the defecated bones from Yam Camp measure in excess of $18 \mathrm{~mm}$. In solomon's sample, however, 108 of bones measure in the vicinity of $20 \mathrm{~mm}$ and some measure up to $40 \mathrm{~mm}$. 


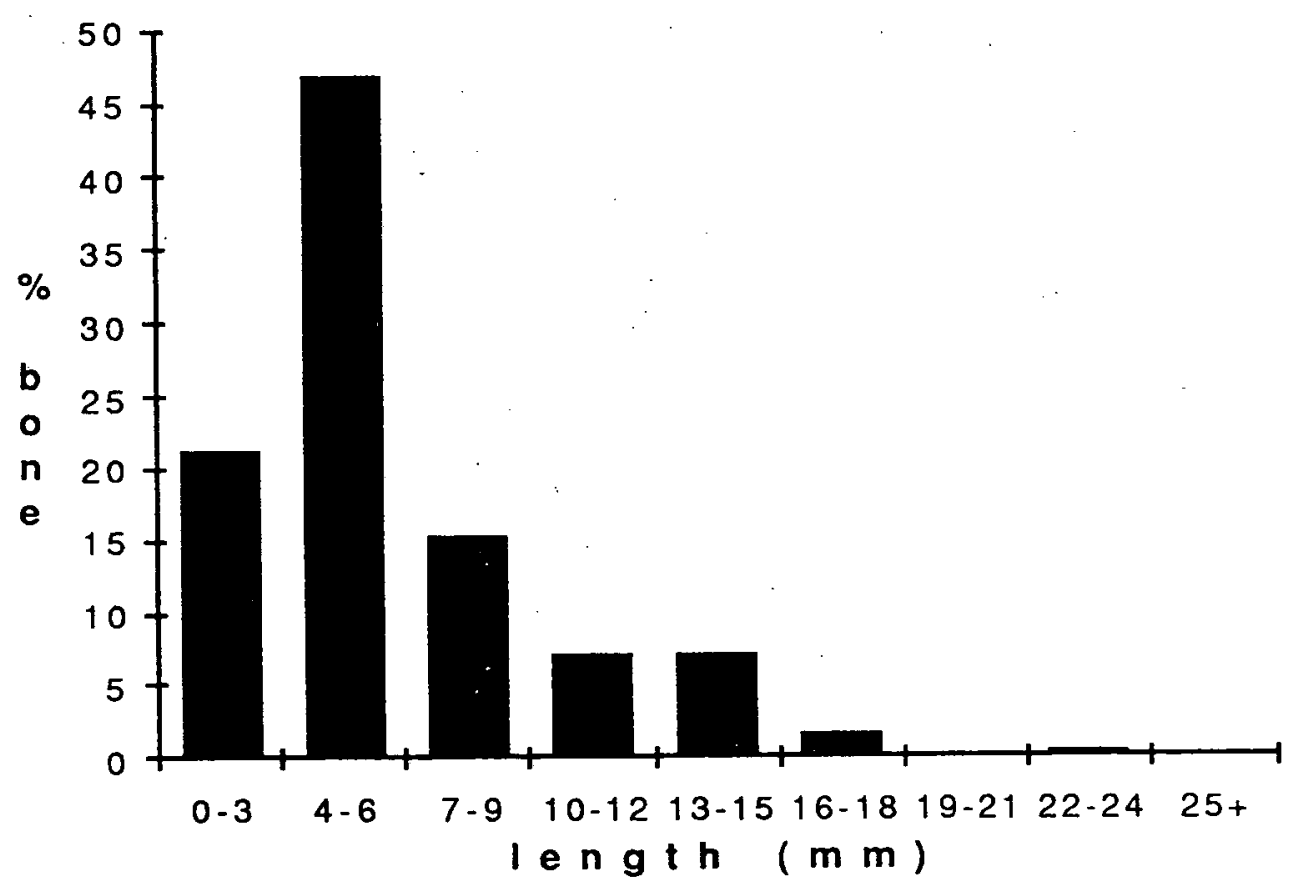

Figure 2. Size of bones from Dingo scats recovered near Yam Camp.

I therefore take the Yam camp data set to be a more realistic estimate of range and frequency in size categories for wild dingoes than that generated by solomon's feeding experiments. Nevertheless, the results presented here do not belittle hér research as both sets of data are complementary and demonstrate that the size of defecated bones correlates with the size of ingested prey. This variability may be related to differences in thickness of bone walls, particularly in the case of long bones. Depending on wall robustness, thickness may affect the minimal size to which bone pieces can effectively be reduced through chewing. Although the testing of this proposition lies beyond the scope of this study, it may be a rewarding avenue of future inquiry.

The implications of the results obtained from naturally-fed dingoes to the Yam Camp archaeological sample now becomes clear. As there is limited overlap in size between the two samples, it is unlikely that the bulk of the archaeological sample was derived from dingo scats. This conclusion is further supported in light of data concerning long bone breakage patterns.

\section{Breakage Patterns of long bones}

The heavy fragmentation of the Yam Camp bones attracted attention for detailed study in the hope that the cause of such fragmentation might be established. One of the avenues explored was the detailed analysis of patterns of breakage of long bones. Long bones in this study refer to limb bones (fibulae, humeri, etc.), metapodials and ribs.

The breakage pattern study involved the analysis of end-breakage patterns and of longitudinal breaks resulting in the collapse of shafts. Four categories were used to classify end breaks as follows:

1. $90^{\circ}$ breakage perpendicular to the long axis of the shaft.

2. $45^{\circ}$ breakage oblique to the long axis of the shaft (commonly referred to by writers as spiral fracture (e.q., see shipman 1981: Figure 5.2). 
3. $V$ breakage resulting in a protruding and bevelled end.

4. Inverted-V breakage resulting in two protruding edges opposite to one another on the same end of the shaft.

Whenever fractures did not fall neatly within one of these four categories, they were classified under the most closely fitting type.

The frequency distribution of breakage types for the long bones of the Yam Camp assemblage is shown in Table 5, as are breakage patterns recorded for the bone sample extracted from the two dingo scats collected above the Yam Camp shelter.

Table 5. Breakage patterns of ends of long bone shafts from Yam Camp Dingo scats and the archaeological assemblage.

\begin{tabular}{lccrcrrrrr}
\hline PROVENANCE & \multicolumn{2}{c}{$90^{\circ}$} & \multicolumn{2}{c}{$45^{\circ}$} & \multicolumn{2}{c}{ V } & \multicolumn{2}{c}{ INVERTED V } & TOTAL \\
& N & $(8)$ & N & $(8)$ & N & $(8)$ & N & $(8)$ & N \\
\hline Yam Camp & 88 & $(59.9)$ & 46 & $(31.3)$ & 10 & $(6.8)$ & 3 & $(2.0)$ & 147 \\
Dingo scats & - & - & 5 & $(45.4)$ & $3(27.3)$ & $3(27.3)$ & 11 & \\
\hline
\end{tabular}

Results show a marked difference in terms of the frequency in $90^{\circ}$ breaks between the two samples. Whereas the excavated sample contains almost 608 of such breaks, the dingo assemblage from Yam Camp contains none. Although the dingo sample is extremely small, the lack of transverse or perpendicular fractures can be taken as diagnostic of long bones defecated by dingoes and, more generally, of bones fractured while fresh. Solomon's work gives support to this contention. As seen in two photographs taken by solomon (1985: Plate 13 A \& B), the long bones show a general lack of $90^{\circ}$ fractures of their ends and a high representation of the three other kinds of fractures.

The high frequency of $90^{\circ}$ fractures in the Yam Camp assemblage may best be explained in terms of breakage having occurred while bone was dry. As explained by shipman (1981:173), "dried bones show a greater tendency [than green bone, that is] to shear perpendicular to the long axis of the bone and its collagen fibres", resulting in a high frequency of columnar fractures (ie. rectangular fragments whose long axes follow the long axis of long bones). In contrast, the characteristics of endbreakage of long bones found in the dingo scats are typical of fractures sustained while bone was green or fresh. That is, all three types of breaks recorded are set oblique to the long axis of the bone, being $45^{\circ}$ breaks, $V$ or inverted-V breaks. Such breaks are known as spiral fractures $\left(45^{\circ}\right.$ break) and jagged fractures (V and inverted-V breaks), both of which are mostly commonly recorded for fresh bone.

There is also a marked difference between the two bone samples in respect of the topography of the fractures present on long bone ends. In the case of the excavated bone, smooth fractures predominate (especially transverse breaks), whereas such features are lacking on bones processed by the Yam Camp dingo. Instead, the defecated long bones bear saw-tooth fractures which are common to bone processed by dingoes as well as a wide range of other predators (including humans) and natural agents. In short, this kind of fracturing is common to virtually all bone broken while fresh (Binford 1981; Shipman 1981). 
As taphonomic research progresses; it becomes increasingly obvious that smooth perpendicular fractures generally occur on long bones which have undergone important chemical and mechanical structural change. This occurs through pronounced burning (which commonly produces square fragments - see Shipman 1981:177; Solomon 1986) or through natural decay during fossilization which results in the break-down of collagen fibres. The outcome of such alterations is a smooth fracture which follows the length of fibres or runs perpendicular to them (see Shipman 1981).

On the basis of the high frequency of smooth transverse fractures on long bones at Yam Camp, I infer that chemical processes played an important role in producing the bulk of the fragmentation present in the assemblage. Another possible cause of this breakage which was considered was exposure to intense or prolonged heat, both of which produce distinctive breakage patterns (David 1987). However, this explanation was rejected as a relatively low percentage of the fragmented bone shows evidence of calcination (see Table 3) (for details on colouring resulting from exposure to heat, see David 1987).

Data available concerning collapsed bone shafts further supports the proposition that fragmentation was caused by the natural process of structural break-down. Of a sample of 77 shafts, 65 (84.48) are collapsed. In contrast, only one-third of the long bones found in the dingo scats were collapsed. It is thus clear that dingo can be excluded as the agent responsible for the collapsing of the Yam Camp long bones.

As Aborigines are reported to have smashed bones to extract marrow (Dawson 1881:18; Gould 1979; Hayden 1979:141-166; Meehan 1982:147), a human agency was also considered for the collapsed shafts. However, this hypothesis was rejected for two reasons. Firstly, none of the bone exhibited definite percussion marks conforming to characteristics derived from bone marrow extraction experiments (Solomon 1985:71). secondly, given the bone size data, it can be asserted that many of the longitudinally split Yam Camp long bones would have contained too little marrow to warrant the effort required to smash their thick walls (see Yellen 1977:8).

The only remaining explanation which may account for the collapse of this large proportion of long bones is natural deterioration of the collagen fibre. This explanation is strengthened by the fact that most longitudinal breaks are clean and clearly parallel to the longitudinal bone fibres. These traits are indicative of natural fracture induced during or following the process of bone fossilization (Shipman 1981 ).

In short, I infer that the patterns of long bone breakage from Yam camp indicate that the heavy fragmentation of the bone assemblage largely took place after burial of the bones as a result of natural deterioration of the bone structure. It is also probable that other factore accelerated the fragmentation process; I suggest trampling in particular. Thus, at the time of deposition, bones would have been on average much larger than those contained in the faeces of wild dingoes. I am confident that the bulk of the bone assemblage from Yam Camp was not the result of dingo defecation.

\section{Shape of Bones from Yam Camp}

Another useful means to study the nature of taphonomic processes operating at Yam Camp is the consideration of shape of all types of bones present in the assemblage. Accordingly, I have developed nine 
categories of bone shape including: rectanqular (Re), trianqular (Tri), trapezoid (Tra), splinter (Spli), lozenge (Lo), oval (Ov), square (Sq), round (Rou) and others (0).

Most of these shape categories need not be defined as they are self-evident. Splinter refers to a piece which is at least five times as long as it is wide. Other incorporates shapes not represented by one of the other eight. Commonly, candidates for others are either fragments with odd shapes (e.g. crescent) or represent whole bones (most of which do not assume standard geometric shapes). In cases where bones did not neatly fit one of the eight shape categories, they were classified under the most closely affiliated shape.

Figure 3 illustrates the frequency distribution of shape types for both the archaeological bone sample and the dingo scat sample and indicates a number of interesting differences between the two assemblages. First, rectangular and square shapes are far more frequent in the archaeological sample than in the dingo sample. Second, oval and round shapes are in much more frequent in the dingo scats sample. Third, the category others is much more represented in the dingo collection.

\section{Yam Camp assemblage $\square$ Bone from dingo faeces}

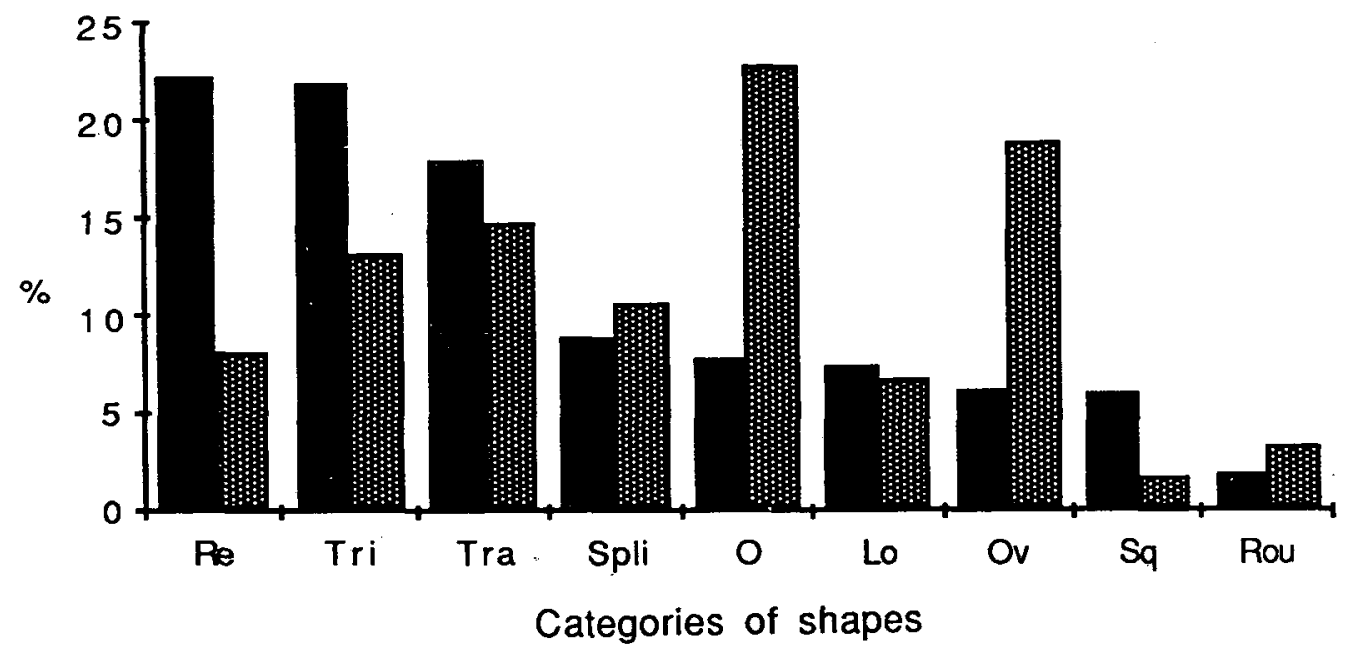

Figure 3. Shape of bones from Yam Camp compared with defecated bone.

These differences may be explained in terms of differing attritional agents. The high frequency of round and oval shapes present in the dingo scat sample may best be explained in terms of the effects of gastric juices on certain bones or bone parts (e.g. scapula, ends of long bones) which are highly susceptible to chemical erosion through exposure to such digestive secretions. The relatively high frequency of the category other in the dingo sample reflects the resilience of some bones (such as phalangae) to digestion. As a result, they preserve their original shape which, in most cases, can only be classified in the category other. The high frequency of angular bone (rectanqular and square) in the Yam Camp assemblage may best be explained in terms of natural processes, i.e. as a result of chemical break-down of structure.

In sum, the shape analysis result adds strength to the proposition that the contribution to Yam Camp bone assemblage by dingoes is negligible. 
Little attention has so far been given to other agents which may have contributed to the modification of individual bones as well as to the overall content of the assemblage. This question is now addressed on the basis of a microscopic examination of the bone.

Bones were examined under low power microscopy to identify marks which were not normal natural features. All such marks were recorded except for both freshly-made ones which probably represent damage sustained during or after excavation and for pits and other marks not forming a distinct pattern and measuring less than $0.1 \mathrm{~mm}$ in width.

The most common animal marks identified are those produced by rodents. All rodents that marked the Yam Camp bones belong to species smaller than the common grey mouse. This inference was based on the width of incisor impressions, the spacing between incisor impressions and the spacing of grooves within tracks formed by individual teeth. The frequency of bone marking by rodents is given in Table 6 .

Table 6. Frequency of occurrence of rodent tooth marks and defecated bone.

\begin{tabular}{lccccc}
\hline SPIT & $\begin{array}{c}\text { SAMPLE SIZE } \\
\text { N }\end{array}$ & $\begin{array}{c}\text { RODENT } \\
\text { N }\end{array}$ & $\begin{array}{c}\text { MARKS } \\
8\end{array}$ & $\begin{array}{c}\text { DEFECATED BONE } \\
\text { N }\end{array}$ & $\begin{array}{c}\text { 8 } \\
\end{array}$ \\
\hline 1 & 96 & 5 & 5.2 & 2 & 2.1 \\
2 & 51 & 2 & 3.9 & 2 & 3.9 \\
3 & 42 & 1 & 2.4 & - & - \\
4 & 188 & 3 & 1.6 & 2 & 1.1 \\
5 & 51 & 2 & 3.9 & - & - \\
6 & 40 & - & - & - & - \\
$7-18$ & 10 & - & - & - & - \\
\hline
\end{tabular}

The proportion of bone gnawed by rodents is relatively low throughout the Yam Camp sequence. The absence of rodent marks on bones from lower spits may be due to small sample size rather than to the absence of rodents per se. The evidence also indicates that while rodents scavenged bones at the site, they did not introduce bones into the archaeological deposits. This is simply inferred by considering that these large bones far out-weighed the rodents (which probably weighed less than $5.0 \mathrm{~g}$ ). All rodent-produced marking (except in three cases) is characterised by the presence of perpendicular and/or oblique incisions combined with chipping back of the gnawed edge.

Digestion and defecation marks are the second most common type of marks represented. Their occurrence is very low, only six bones (18) displaying such marks (Table 6). All display at least two of the following traits: pitting, surface polishing, edge smoothing and adhering faecal matrix. Specific identifications include a maxillary fragment of a white-tailed rat (Uromys sp.), the vertebra of a small species of skink, the articulating proximal end of a possum humerus (Trichosurus vulpecula) and a skull fragment of a large macropod. The rat and skink remains accord with the hunting patterns of dingoes and quolls. However, the possum and large macropod remains are more likely to represent human hunting. It is therefore probable that at least some of the defecated bone comes from an animal that either scavenged human refuse or was fed by humans (esp. dingo). 
Weathering cracks, exhibited on a single bone fragment, represents the only other type of distinctive marking found in the bone assemblage. This rare example of weathered bone from almost 500 examined indicates that the majority of Yam Camp bones were quickly incorporated into the site's sediments.

A total of 49 bones exhibited other marks (some displaying two or more types) which may be summarised as follows:

1. Twenty-six bones bear incisions commonly arranged as sets of parallel grooves mostly perpendicular or oblique to the long axis of the bones. The grooves are almost always very narrow (about $0.1 \mathrm{~mm}$ or less).

2. Twenty-two bones are pitted. Pits range between less than $0.1 \mathrm{~mm}$ to 1.Omm in diameter. They are commonly circular, although other shapes are present. These pits can not be interpreted as tooth marks since they are too small and do not appear "crater-like" as one would expect from tooth marks made on fresh bone.

3. Fifteen bones have edge-damage appearing as series of notches similar to trimmed edges of stone artefacts. However, chip scars on the Yam Camp bones are generally smaller than those usually produced on stone artefacts, being for the most part $1 \mathrm{~mm}$ wide or less. These marks do not apparently represent use-wear because no other features are present on the bone that would be expected had the bones been used as tools (e.g. edge-blunting, striae and gloss; see Kamminga 1982). They possibly represent damage resulting from trampling.

4. Thirteen notched bones were recorded; none appear to have been made by teeth as they are too small.

5. Two crenulated edges were found (for definition, see Binford 1981:44 guoted in David 1984:48) but there was no definitive evidence to suggest they were produced by carnivore teeth (the crenulation is too fine).

6. Two punctures were identified that can not be interpreted with any degree of certainty as representing animal activity.

7. Two shaving marks, possibly the result of tool use.

8. One unusual and highly distinctive series of overlapping peckings, the patterns of which require more detailed examination before their origin may be assessed.

Since none of the above-listed marks clearly represent activities of carnivores and/or other natural agents and none clearly exhibit human butchering, eating or tool-use, it is plausible that the bulk of them were sustained as a result of humain trampling. This hypothesis is not tested here, primarily because at present there is insufficient data relevant to the issue for Yam Camp.

Other Agents Possibly Affecting the Bone Assemblage

Several taphonomic aspects have yet to be considered for the bone assemblage. One concerns the possibility that termites caused damage to the bones. Termites are common in the region and a large termite mound housing a colony of coptotermes acinaciformis presently stands about $2 \mathrm{~m}$ away from the excavation area. A termite presence has also been noted by Watson and Flood (1987) at Echidna shelter, Green Ant shelter 1 and 
other sites on the Koolburra Plateau, some 50km North-West of the Yam Camp site. Although . acinaciformis is one of the largest species of termites in Australia, experiments have shown they cause only sight damage to bones. Watson and Abbey (1986:250) noted:

"The most severe damage to compact bone was assessed as slight attack [...]. Cancellous bone was damaged more extensively [...]. The greater effect on cancellous bone was possibly an "edge effect" [...] comparable to the rounding of cut edges and along cracks in dense bone."

A close examination of the bone from Yam Camp leads me to argue that $C$. acinaciformis termites have either not damaged the bones or, if so, damage is very limited and represented on compact bone in the form of pits (found on 22 bones) and possibly fine crenulation of edges (present on two bones). It is unlikely that termites caused the destruction of bones at the site.

Another possibility was that this termite species may have significantly disturbed the archaeological deposits in the course of their tunnelling and mound-building activities (see cloud, Gustafson and Watson 1980). This is now thought unlikely in view of observations made at Echidna shelter and Green Ant shelter 1 :

"What is surprising is that their tunnelling activities do not appear to have disturbed the deposits from the archaeological point of view, for there were no signs of disturbance nor any inversions in the series of radiocarbon dates at either excavated site, nor was there evidence of major damage to bone deposits" (Watson \& Flood 1987:25).

Owls or other birds of prey were also considered as possible contributors of bones to the site as there are several rock ledges within the shelter that would provide ideal roosts. One of these overlooks the excavation area. However, there are no apparent signs of roosts and there is no direct evidence of such activity in the Yam camp bone assemblage. Bone contribution by owls and other birds of prey is commonly assessed on the basis of the presence of small animals, (especially rodents, lizards and small bandicoots), the relative frequencies of anatomical elements and the bone condition (e.q. Archer and Baynes 1972; Hope 1973:5-7; Baynes et al. 1976:103; Bowdler 1979:163; Shipman 1981:132; Marsh́all 1986).

Although a detailed analysis of the fauna has not yet been carried out, the material so far examined indicates that very few animals could have been brought in by birds. These include a small bandicoot (possibly of the genus perameleg), two species of rodents and two species of reptiles. Their remains are limited, representing less than $30 \%$ of the MNI so far established and 178 of the minimum number of species identified. There is, however, no conclusive evidence that any such animals were deposited by predatory birds. None of the bones exhibit an adhering matrix of pellets. It is not possible to assess bird contribution on the basis of the representativeness and condition of anatomical elements since few bones can be identified due to the heavily fragmented nature of the assemblage. This fragmentation may in fact offer evidence that birds of prey did not deposit the bones. Had bones been deposited as bird pellets, they should be less fragmented than other bones in the assemblage as they would be protected against chemical and mechanical damage by the pellet matrix. Thus, if birds of prey did contribute to 
the deposition of Yam Camp bone, they did so in a very limited way. It is therefore unlikely. that their minor contri-bution would cause a serious analytical problem to the reconstruction of the economic sequence at the site.

Similarly, the contribution that dasyurids (quolis) may have made to the assemblage is not regarded as significant because of the limited number of species and individuals that could have been brought in by these animals. Perhaps more problematic would be the potential damage that dasyurids can make to bones discarded by humans. If quolls scavenge human refuse they could introduce serious bias in the economic reconstruction as it is likely that such scavenging would lead to the selective destruction of particular species, especially medium and small animals.

In summary, in the Yam Camp case, I am confident that scavenging by quolls and other carnivores such as dingo did not occur or, if so, it was a very occasional event. This inference is based on the facts that not one definite tooth mark has been identified, bones were rapidly incorporated into the sediments (indicated by the general lack of weathered bone) and there is a relatively high frequency of spongy bone represented in the assemblage (this type of bone is prone to destruction by predators; see Binford and Bertram 1977; Solomon 1985).

Water transport is the only non-biogenic process considered as a possible modifier of the assemblage composition. Rainfall is very high in the region and the shelter is located on a slope, the gradient of which would generate sufficient water velocity to transport bone either to the site or away from it. The archaeological data, however, negates this possibility for three reasons. Firstly, there is ample evidence to indicate excellent organic preservation at Yam Camp. Although the bones underwent important chemical changes which resulted in increased fragmentation through time, this apparently did not lead to significant losses in bone mass at the site. This is suggested by the pH values of the sediments, data on the sharpness of bone edges, and correlations in the relative frequency of lithic and various organic materials according to depth. Secondly, the bones have not been sorted as one would expect as a result of water transport. This fact is best demonstrated by the bone size data which show a range of sizes represented at Yam Camp. Thirdly, the wide range of shapes represented is not characteristic of water-transported bone assemblages (see Shipman 1981:26-41).

\section{SURASARY OF THE TAPHONOMIC HISTORY OF YAM CAMP AND IMPLICATIONS}

The taphonomic history of the Yam camp rockshelter may be summarised as follows:

1) Dingoes, quolls and other medium-to-large non-human predators have had an insignificant effect on the composition of the faunal assemblage.

2) Bones were rapidly incorporated. into the sediments as indicated by the general lack of weathering marks.

3) The bulk of the fragmentation sustained by the bones occurred while they were dry or old and after they were incorporated into the sediments. Fragmentation resulted from a change in the chemical composition of the bone. Breakage was probably accelerated by human trampling. 
4) Rodents did gnaw on a small proportion of the bone but these activities caused only minor edge damage and did not lead to the complete destruction of any of the bones. The very small size of these rodents $(<5.0 \mathrm{~g})$, as inferred from the nature of the tooth marks, also indicates that these rodents would have been incapable of either bringing large bone to, or removing bone from, the site.

5) Owls and other birds of prey made apparently no contribution to the faunal assemblage.

6) There is no unequivocal evidence of bone damage resulting from termite attacks, although it is possible that a small portion of the bones bear such marks in the form of pitting and fine edge-crenulation. If termites did cause damage to some of the bones, this would have been minimal and would not have destroyed any bones.

7) There is no evidence to suggest that water action introduced, destroyed or removed any bone.

Finally, on the basis of the above study I feel confident that the faunal assemblage from Yam Camp now offers a more reliable basis for the reconstruction of economic and other cultural aspects than had this study not been carried out. The study has indicated that the bulk of the bone was clearly deposited as a result of human behaviour and that potential attritional agents that might have modified the assemblage composition have either not affected the bone at all or affected it in a very limited manner. There is no evidence that selective destruction or removal of bones took place that would lead to bias in terms of species representation, skeletal elements or even their parts (such as compact or spongy bone). In short, preservation of the bone assemblage is excellent, although it has undergone size reduction through fragmentation resulting from chemical deterioration of collagen fibres.

At a more general level, these results demonstrate the importance of reconstructing the taphonomic history of a site prior to cultural analysis. Agents of deposition and those which modified the archaeological assemblage should be understood if we are to make reliable inferences about past human behaviour and cultural processes.. This study also demonstrates the archaeological potential of unidentifiable bone fragments present in faunal assemblages. As shipman (1981:128) correctly pointed out:

\footnotetext{
"Nearly all of the literature and most collectors fail to appreciate another source of paleoecological and taphonomic information in any fossil assemblage: the indeterminate fragments."
}

\section{ACKNOWLEDGMENTS'S}

Several deserve acknowledgment: I thank in particular Mike Morwood for advice and constructive comments on several drafts of this paper and for allowing me to become involved with his S.E. Cape York Archaeological Project; Kim Gollan for discussing taphonomic aspects; Jane Balme for guidance regarding analytical approaches; and Malcolm Abel for providing me with scats of wild dingoes, laboratory assistance and for sharing his extensive knowledge of bones. The advice and detailed comments provided by an anonymous $Q A R$ referee are also acknowledged. Thanks to $\mathrm{Di}$ Watson for help with word processing matters. Finally, I wish to thank Jay Hall for his advice and editorial help. 


\section{REFERENCES CITED}

Abel, M. 1989 Jowalbinna mammals - preliminary report. Unpublished report. Department of Archaeology and Palaeoanthropology, University of New England, Armidale.

Archer, B. and A. Baynes 1972 Prehistoric mammal faunas from two small caves in the extreme south-west of Western Australia. Journal of the Royal Society ${ }^{\circ}$ of Western Australia 55:80-89.

Baynes, A., D. Merrilees and J.K. Porter 1976 (nominally 1975) Mammal remains from the upper levels of a late Pleistocene deposit in Devil's Lair, Western Australia. Journal of the Royal Society of Western Australia 58(4):97-126.

Binford, L.R. 1981 Bones: Ancient Men and Modern Myths. New York: Academic Press.

Binford, L.R. and J.B. Bertram 1977 Bone frequencies - and attritional processes. In L.R. Binford (ed.), For Theory Building in Archaeology : Essays on Faunal Remaing, Aquatic Resources, Spatial Analysis, Systemic Modeling, pp. 7.7-153. New York: Academic Press.

Bowdler, S. 1979 Hunter Hill, Hunter. Island. Unpublished Ph.D thesis. Department of Prehistory, The Australian National University, Canberra.

Cloud, P., L.B. Gustafson and J.A.L. Watson 1980 The works of living social insects as pseudofossils and the age of the oldest know Metazoa. Science 210:1013-1015.

David, B. 1984 Man Vs. Dingo: The Identification of Bone Remains from Archaeological Sites, with Specific Reference to Walkunder Arch Cave, Chillagoe Northeast Queensland. Cultural Resource Management Monograph Series No. 5 Archaeology Branch, Brisbane.

David, B. 1987 Animals have to die sometime, or is burnt bone burnt. Paper presented at the Taphonomy of Bones Conference, 21st-22nd February 1987, University of New England, Armidale.

Dawson, J 1881 Australian Aborigines. George Robertson, Melbourne.

Gould, R.A. 1979 Exotic stones and battered bones. World Archaeology $32: 29-37$.

Hayden, B. 1979 Paleolithic Reflections. Australian Institute of Aboriginal Studies, Canberra.

Hope, J. 1973 Appendix XIV - Analysi.s of bone from cloggs Cave, Buchan, N.E. Victoria. In J. Flood, The Moth Hunters. Unpublished Ph.D thesis, Australian National University, Canberra.

Kamminga, J. 1982 Over the Edge: Functional Analysis of Australian Stone Tools. Occasional Papers in Anthropology, No. 12. Anthropology Museum, University of Queensland, St. Lucia. 
Marshall, B. 1986 An experimental evaluation of the criteria used to distinguish owl-deposited bone in archaeological cave deposits in Australia. Australian Archaeology 22:104-121.

Meehan, B. 1982 Shell Bed to Shell Midden. Australian Institute of Aboriginal Studies, Canberra.

Morwood, M.J. 1989 The archaeology of Aboriginal art in S.E. Cape York: preliminary report on the 1989 fieldwork. Rock Art Research $6(2): 155-156$.

Morwood, M.J. 1990 Preliminary report on the 1989 excavations at Yam Camp rockshelter, Red Bluff rockshelter, Magnificent rockshelter and Sandy Creek rockshelter. Unpublished report to the Quinkan Reserves Trust.

Pearson, พ. 1989 A technological analysis of stone artefacts from Yam Camp surface scatter and rockshelter, S.E. Cape York. Queensland Archaeological Research 6:91-102.

Shipman, P. 1981 Life History of a Fossil. Cambridge: Harvard University Press.

Solomon, S. 1985 People and other aggravations: taphonomic research in Australia. Unpublished BA(Hons) thesis. Archaeology and Palaeoanthropology Department, University of New England, Armidale.

Solomon, S. 1986 Appendix 2: A taphonomic analysis of the fauna from Gatton rock shelter (squares $\mathrm{G} 4 \mathrm{C}$ and $\mathrm{G} 4 \mathrm{~d}$ ). In M.J. Morwood, The Archaeology of art: Excavations at Maidenwell and Gatton Shelters, Southeast Queensland. Queensland Archaeological Research 3:88-132.

Watson, J.A.I. and H.M. Abbey 1986 The effects of termites (Isoptera) on bones: Some archeological implications. Sociobiology $11(3): 245-254$.

Watson, J.A.I. and J.M. Flood 1987 Termite and wasp damage to Australian rock art. Rock Art Research $4(1): 17-28$.

Yellen, J.E. 1977 Archaeological Approaches to the Present: Models for Reconstructing the Past. New York: Academic Press. 